\title{
The effect of zinc supplementation on diarrheal diseases in children in the Niger Delta Sub-Region of Nigeria
}

\author{
Eme Asuquo ${ }^{1}$, Udeme Georgewill ${ }^{2^{*}}$, Idorenyin $\mathrm{Nta}^{1}$, Nkechinyere Enyidah $^{1}$, Essiet Umofia ${ }^{1}$, \\ Suanu Deekae ${ }^{1}$ \\ ${ }^{1}$ Department of Preventive and Social Medicine, College of Health Sciences, University of Port Harcourt, Port Harcourt, Nigeria \\ ${ }^{2}$ Department of Pharmacology, College of Health Sciences, University of Port Harcourt, Port Harcourt, Nigeria; \\ *Corresponding Author: udgeorgewill@yahoo.com
}

Received 5 January 2012; revised 8 February 2012; accepted 12 March 2012

\section{ABSTRACT}

This study evaluated the trend of diarrheal diseases managed with zinc supplementation by comparing it with diarrheal diseases managed without zinc supplementation at the University of Port Harcourt Teaching hospital (UPTH), Nigeria. The study was a descriptive retrospective study done at the Diarrhea Training Unit (DTU) of UPTH to determine the effect of zinc supplementation in the management of diarrhea in children under 5 years. Out of the 134 case records studied, of children aged 0 to 59 months who presented with diarrhea at the DTU, 57 children did not receive zinc supplementation between October and December, 2007 and 77 children received zinc supplementation between October and December 2009. The results showed that (74) $96.1 \%$ of patients who received zinc supplementation, and (48) $84.2 \%$ of those who did not, had no repeat diarrheal episodes when seen at the follow up clinic. On the other hand, $1.3 \%$ of those who received zinc supplementation and $1.8 \%$ of those who did not, had increased episodes of diarrhea when seen at the follow up clinic. Of those who received, and those who did not receive zinc supplementation, $2.6 \%$ and $14 \%$ respectively, had reduced episodes of diarrhea. These findings clearly demonstrate the effectiveness of zinc supplementation in halting the course of diarrheal diseases in children aged 0 to 59 months. We therefore advocate for the use of zinc supplementation in the management of diarrhea in Nigeria.

Keywords: Diarrheal Diseases; Zinc

Supplementation; Children; Niger Delta Sub-Region

\section{INTRODUCTION}

Diarrhea is defined as the passage of three or more stools in a day of consistency softer than usual for the child or one watery stool per day [1]. There are four clinical types of diarrhea namely: acute watery diarrhea which lasts several hours or days and can result in dehydration and weight loss if feeding is not continued; acute bloody diarrhea, also called dysentery and can result in intestinal damage, sepsis and malnutrition, other complications and including dehydration; persistent diarrhea, which lasts 14 days or longer and can result in malnutrition and serious non-intestinal infection including dehydration may also occur; diarrhea with severe malnutrition (marasmus or kwashiorkor) the resulting in severe systemic infection, dehydration, heart failure and vitamin and mineral deficiency [2-5]. Diarrheal disease poses a significant public health problem on a global scale especially in developing countries and it is the second leading cause of death in children under five years globally [2]. Mortality due to diarrhea can be reduced by interventions like oral rehydration therapy, optimal breast feeding practices, improved nutrition, increased access to clear water and sanitation facilities, improved personal and domestic hygiene and appropriate drug therapy where necessary [3].

In developing countries, diarrhea causes around two million child deaths annually and has been recognized as one of the major killer diseases in childhood which can be readily prevented by some inexpensive interventions and is therefore a public health challenge especially in developing countries. It kills more young children than AIDS, malaria and measles combined [1]. India, Nigeria, China, Pakistan, Democratic Republic of Congo and Ethiopia accounts for $50 \%$ of deaths under age five [3]. In Nigeria, $24 \%$ of infant mortality rates (IMR) and $19 \%$ of under-five (U-5) deaths are due to diarrheal diseases [6]. Overall, children experience an average of 3.3 epi- 
sodes of diarrhea per year, and in areas where the episodes are frequent, young children spend more than 15\% of their childhood with diarrhea [7]. Children make up $40 \%$ of the population of developing countries. In the National Demographic and Health survey in 2008, it was found that in Nigeria, forty-five percent of household members are children under the age of 15 [8]. One hundred and fifty-seven children per 1000 live births, or about 1 child out of every 6 , die before reaching age five [8]. During the two weeks before the survey, 1 in $10 \mathrm{Ni}$ gerian children under-five had diarrhea. The rate was highest (16\%) among children 12 - 23 months [8]. Diarrhea accounted for $18 \%$ of under-5 deaths [9].

Zinc supplementation could help reduce the duration and severity of diarrhea, and is recommended by the World Health Organization and UNICEF [10]. Children in developing countries are often zinc deficient and therefore zinc supplementation is a possible treatment for diarrhea though it can have adverse effects if given in high doses. Zinc deficiency is widespread among children in developing countries and occurs in most parts of Latin America, Africa, the Middle East, and South Asia. [11] It is due to lack of intake of animal food, high dietary phytate content, inadequate food intake and increased faecal losses during diarrhea and is exacerbated by the net loss of zinc during diarrhea [10]. Additional studies have shown that a short course supplementation with zinc (10 - $20 \mathrm{mg}$ per day for 10 to 14 days) reduces the incidence of diarrhea for 2 to 3 months.

A meta-analysis of five studies of zinc treatment for acute diarrhea found a summary estimate for reduction in duration of $16 \%$. Possible mechanisms for the effect of zinc treatment on the duration of diarrhea include improved absorption of water and electrolytes by the intestine, faster regeneration of gut epithelium, increased levels of enterocyte brush border enzymes, and enhanced immune response, leading to early clearance of diarrheal pathogens from the intestine [2-5,12]. Zinc is important for normal growth and development and for reducing childhood diarrhea cases. Diarrheal prevalence has fallen by $34 \%$ in the past decade. Zinc supplementation during acute diarrhea has been shown to enhance recovery and continuous supplementation studies have shown a significant reduction in the incidence of persistent diarrhea [2-5,12].

There has been a $20 \%$ reduction in duration of acute diarrhea, $25 \%$ reduction in stool output in acute diarrhea, $24 \%$ reduction in duration of persistent diarrhea and $42 \%$ reduction in treatment failure or death in persistent diarrhea [2-5,12].

Based on these studies, it is now recommended that zinc (10 - $20 \mathrm{mg} /$ day) be given for 10 to 14 days to all children with diarrhea [13]. However, the literature on the use of zinc supplementation in the management of diarrheal diseases and its effects on the outcome of diarrheal diseases are non-existent or very scanty in the Niger Delta Sub-region, a region with high prevalence of diarrheal disease. Thus this study compared the trend of diarrheal diseases managed with zinc supplementation and those managed without zinc supplementation in UPTH.

\section{METHODOLOGY}

This was a descriptive retrospective study done at the Diarrhea Training Unit (DTU) of the University of Port Harcourt Teaching Hospital (UPTH), Alakahia, Nigeria. Case records of children aged 0 to 59 months who presented with diarrhea at the DTU between October and December, 2007 and October and December 2009 were used. Case records were used based on the inclusion criteria; case records of children with diarrhea and case records of children aged less than or equal to 5 years. Case records which did not fit into these criteria were excluded. The sample size, consisting of 134 case records was drawn from patients treated with standard Oral Rehydration Solution (ORS) from October to December 2007 (78 patients) and those who had ORS in addition to zinc supplementation from October to December 2009, 57 patients.

\section{Data Analysis}

Data were extracted and analyzed using SPSS version 17.0. Diarrhea duration and severity were the primary outcomes. All results obtained were statistically analyzed and presented as bar charts expressing means.

\section{RESULTS}

The results of the study are presented in Figures $\mathbf{1}$ and 2 below.

\section{DISCUSSION}

In Nigeria, $24 \%$ of infant mortality rates (IMR) and $19 \%$ of under-five deaths are due to diarrheal diseases. Overall, children experience an average of 3.3 episodes of diarrhea per year, and in areas where the episodes are frequent, young children spend more than $15 \%$ of their childhood with diarrhea. Intervention trials indicate that zinc given during acute diarrhea is efficacious in reducing the duration and severity of the illness. Thus, therapeutic use of zinc has the potential of reducing the annual 2.5 million childhood diarrheal deaths.

Follow up findings show that $93.6 \%$ (72) of patients who received and $84.2 \%$ (48) of those who did not receive zinc supplementation had no repeat diarrheal episode when seen at the follow up clinic while $1.3 \%$ and $1.8 \%$ of those who received and did not receive zinc supplementation respectively had increased diarrhea epi- 


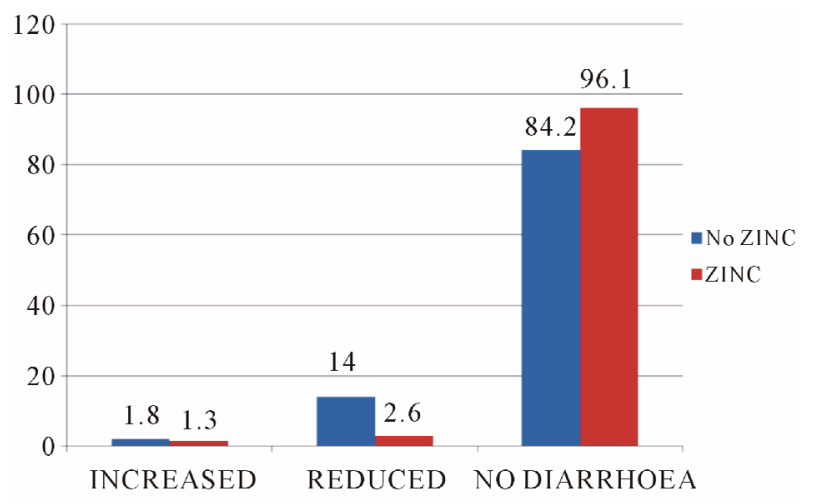

Figure 1. Follow up findings showing reduced incidence of diarrheal episodes.

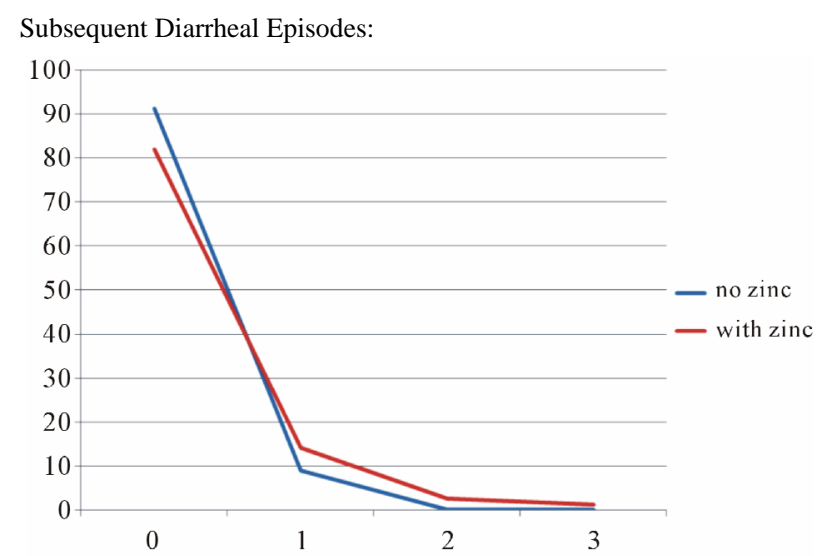

Figure 2. Chart of subsequent diarrheal episodes showing a reduction in diarrheal episodes following zinc supplementation treatment compared to treatment without zinc.

sodes when seen at the follow up clinic. 5.1\% and 14\% of those who received and did not receive zinc supplementation respectively had reduced diarrheal incidence when seen at the follow up clinic as shown in Figure 1. According to the Zinc Investigators' Collaborative Group, Pediatrics, 1999 there has been a 34\% drop in the prevalence of diarrhea in the past decade which is about 3.4\% per year. Our study showed a drop of about $3.6 \%$ per year which is consistent with their findings.

The study further shows that $8.8 \%$ and $18.2 \%$ of those who received and did not receive zinc supplementation respectively had 1 to 3 subsequent diarrheal episodes as shown in Figure 2. This shows a reduction in number of diarrheal episodes following treatment.

Zinc is known to play a role in diarrhea where it reduces the bulk and frequency of diarrhea. It also acts as an immune modulator. The current study showed that majority of the children under-five years who presented during follow up had no diarrhea, although a greater percentage of these had received zinc supplementation, thus demonstrating the effectiveness of zinc supplementation as an adjunct to ORS in the management of diar- rheal diseases. This corroborates the research done in Bangladesh which showed that children who received zinc supplementation during and after diarrhea had shorter duration of diarrhea, 15\% lower incidence of diarrhea, and a trend suggesting fewer diarrhea related hospital admissions. The results of these pooled analyses and additional information from other published randomized trials indicate that zinc, given during acute or persistent diarrhea, can have substantial clinical benefit and suggest that this adjunctive therapy could reduce the risks of dehydration and death from diarrhea [4]. Numerous studies have shown that despite the type of diarrhea, children receiving zinc supplementation had shorter episodes of diarrhea, passed fewer stools per day, and were less likely to have progression to prolonged diarrhea, compared with children receiving placebo [2-5,12] Perhaps the use of this effective and inexpensive nutrient supplement would be helpful in efforts to reduce the now common treatment of diarrhea with unnecessary antibiotics and other drugs [14]. At the same time, it will be important to continue the promotion of appropriate fluid and dietary therapy as the mainstay of efforts to reduce mortality from diarrhea. For children and young children with acute diarrhea, zinc supplementation results in clinically important reductions in the duration and severity of diarrhea [10]. Zinc supplementation decreases the duration and severity of the episode, decreases the need for expensive hospitalization and decreases the use of unnecessary antibiotics and other drugs [13]. Supplementation has a clinically significant beneficial impact on the clinical course of acute diarrhea, reducing the severity and duration of diarrhea [15]. The current study therefore concludes that zinc supplementation treatment of diarrhea significantly reduces the incidence of diarrheal episodes in children $<5$ years in the Niger Delta Sub-Region and is therefore recommended for the treatment of acute diarrheal episodes in children of this Sub-region.

\section{ACKNOWLEDGEMENT}

The authors are grateful to Prof. O.A. Georgewill for his useful contributions.

\section{REFERENCES}

[1] Khan, P.A. (2008) Infectious diseases. Basis of pediatrics. 7th Edition, Zahid Bashir Printers, Lahore, 201-282.

[2] Bhutta, Z.A., Black, R.E., Brown, K.H., Gardner, M.J., Gore, S., Hidayat, A., Khatun, F., Martorell, R., Ninh, N.X., Penny, M.E., Rosado, J.L., Roy, S.K., Ruel, M., Sazawal, S. and Shankar A. (1999) Prevention of diarrhea and pneumonia by zinc supplementation in children in developing countries: Pooled analysis of randomized controlled trials; Zinc Investigators Collaborative Group; Aga Khan 
University Medical Center, Karachi.

[3] WHO (1990) World Health Organization Programme for control of diarrheal diseases; a manual for the treatment of diarrhea. WHO, Geneva.

[4] Bhutta, Z.A., Bird, S.M., Black, R.E., Brown, K.H., Gardner, J.M., Hidayat, A., Khatun, F., Martorell, R., Ninh, N.X., Penny, M.E., Rosado, J.L., Roy, S.K., Ruel, M., Sazawal, S. and Shankar, A. (2000) Therapeutic effects of oral zinc in acute and persistent diarrhea in children in developing countries: Pooled analysis of randomized controlled trials. American Journal of Clinical Nutrition, 72, 1516-1522.

[5] Fischer Walker, C.L. and Black, R.E. (2007) Micronutrients and diarrheal disease. Clinical Infectious Diseases, 45, S73-S77.

[6] Foutaine, O. (2001) Effect of zinc supplementation on clinical course of acute diarrhea; report of a meeting, New Delhi. Journal of Health Population and Nutrition, 19, 338-346.

[7] R. K. Adhikari, N. Bhandari, R. J. Ulvik, K. Mølbak, M. K. Bhan and H. Sommerfelt. (2002) Effectiveness and efficacy of zinc for the treatment of acute diarrhea in young children. Pediatrics, 109, 898-903. doi:10.1542/peds.109.5.898

[8] Al-Sonboli, N., Gurgel, R., Shenkin, A., Hart, C.A. and Cuevas, L. (2003) Zinc supplementation in Brazilian children with acute diarrhea. Annals of Tropical Paediatrics, 23, 3-8. doi:10.1179/000349803125002797

[9] Centre for Health and Population Research (2001) Effect of zinc supplementation on clinical course of acute diarrhea. Journal of Health, Population and Nutrition, 19, 338346.

[10] World Health Organization/United Nations Children's Fund (2004) Joint statement: Clinical management of acute diarrhea (who/fch/cah/04.07). United Nation Children Emergency Fund, Geneva and New York.

[11] National Population Commission (NPC) and ICF Macro. (2008) Nigeria Demographic and Health Survey 2008: Key Findings.

[12] Zinc Investigators' Collaborative Group (1999) Prevention of diarrhea and Zinc Investigators' Collaborative Group, Prevention of diarrhea and pneumonia by zinc supplementation in children in developing countries: Pooled analysis of randomized controlled trials. Journal of Pediatrics, 135, 689-697. doi:10.1016/S0022-3476(99)70086-7

[13] Fewtrell, L., et al. (2005) Water, sanitation, and hygiene interventions to reduce diarrhea in less developed countries: A systematic review and meta-analysis. The Lancet Infectious Diseases, 5, 42. doi:10.1016/S1473-3099(04)01253-8

[14] Al-Sonboli, N., Gurgel, R., Shenkin, A., Hart, C.A. and Cuevas, L.E. (2003) Zinc supplementation in brazilian children with acute diarrhea. Annals of Tropical Paediatrics, 23, 3-8. doi:10.1179/000349803125002797

[15] Lazzerini, M. and Ronfani, L. (2008) Oral zinc for treating diarrhea in children. Cochrane Database of Systematic Reviews, 3. doi:10.1002/14651858.CD005436.pub2 